\title{
Planetary bow shocks: Asymptotic MHD Mach cones
}

\author{
M. Verigin ${ }^{1,2}$, J. Slavin ${ }^{1}$, A. Szabo ${ }^{1}$, G. Kotova ${ }^{2}$, and T. Gombosi ${ }^{3}$ \\ ${ }^{1}$ NASA Goddard Space Flight Center, Code 696, Greenbelt, Maryland 20771, U.S.A. \\ ${ }^{2}$ Space Research Institute of Russian Academy of Sciences, Profsoyuznaya, 84/32, Moscow, 117997, Russia \\ ${ }^{3}$ University of Michigan, Ann Arbor, MI 48109-2143, U.S.A.
}

(Received August 16, 2002; Revised November 20, 2002; Accepted December 17, 2002)

\begin{abstract}
A direct approach for determining the asymptotic MHD Mach cone is formulated and solved. An implicit analytical solution enables the calculation of the asymptotic downstream slope of MHD Mach cones at any clock angle for arbitrary $M_{s}, M_{a}$, and $\vartheta_{b v}$. The solution obtained includes all previously known symmetric cases. The elongation and shift of the asymptotic fast mode shock cross-section are studied for a wide range of upstream plasma parameters as well as its unusual 'chopped' shape under certain conditions. Our results may be useful for planetary shock modeling and MHD numerical codes verification.
\end{abstract}

\section{Introduction}

There is an abundance of physical processes, operating in the vicinity of the planets that are influenced by their bow shocks. Information on the instantaneous position of this boundary is important for studies of the physical processes accompanying electron and ion foreshock formation; charged particle acceleration; the mechanisms for solar wind deceleration in the shock foot and shock itself; analysis of plasma turbulence generation inside the magnetosheath, etc.

Modern 3-D shock models (e.g., Slavin et al., 1984; Khurana and Kivelson, 1994; Bennet et al., 1997; Verigin et al., 1999, 2001) require theoretical guidance regarding the asymptotic, downstream behavior of planetary bow shock slope (cone) angle $\omega$ as a function of sonic $M_{s}$, Alfvenic $M_{a}$ Mach numbers, and angle $\vartheta_{b v}$ between the interplanetary magnetic field $\boldsymbol{B}$ and solar wind velocity $\boldsymbol{V}$ vectors.

The approaches that are generally used for the construction of the asymptotic downstream Mach cone within the framework of ideal magnetohydrodynamics (MHD) are described in a number of papers and textbooks. They are based either on the examination of the cross-section of a sphere of $V / 2$ radius centered at the $-V / 2$ point with a Friedrichs I diagram for plane MHD waves, or on the construction of tangents to the related Friedrichs II diagram for the waves from a point disturbance (e.g., see figure 4 of Spreiter et al., 1966).

Although the first procedure is apparently straightforward and a cross-section can be found quite easily, it permits direct determination of $\omega$ in only a few specific, symmetric cases (Spreiter and Stahara, 1985) as listed in Table 1. For other cases the difference between the direction from the Mach cone axis to any point on the shock surface in the plane perpendicular to cone axis and projection into the same plane of the vector normal to the shock $\boldsymbol{n}$ at that point must be taken

Copy right (C) The Society of Geomagnetism and Earth, Planetary and Space Sciences (SGEPSS); The Seismological Society of Japan; The Volcanological Society of Japan; The Geodetic Society of Japan; The Japanese Society for Planetary Sciences. into account. This fact was often not properly recognized in planetary bow shock modeling.

The second approach is free from the above mentioned difficulty, but requires a complicated construction to obtain the tangent surface to the Friedrichs II diagram for which the only parametric expressions are available either in Cartesian (e.g., Jeffrey, 1966) or in spherical (e.g., Whitham, 1974) coordinates. Neither approach provides a suitable analytic expression for the MHD Mach cone cross-section and $\omega$ for arbitrary $M_{s}, M_{a}$, and $\vartheta_{b v}$.

In the present paper we will present a new method for the determination of asymptotic MHD Mach cones, which will be solved to yield exact analytic expressions convenient for space applications, and we will discuss some specific features of this boundary.

\section{Formulation of the Problem and Solution 2.1 Deduction of Mach cone equation}

Figure 1 depicts the main geometrical notations used throughout the paper. In our reference frame the $X$ axis is antiparallel to the upstream undisturbed flow velocity $\boldsymbol{V}$, while the $Y$ axis is placed in the $\boldsymbol{B}, \boldsymbol{V}$ plane, with undisturbed upstream interplanetary magnetic field line passing through the origin, II-nd, and IV-th quadrants of the reference frame. This coordinate system corresponds to the GIPM reference frame used to study planetary bow shock related phenomena (Peredo et al., 1995).

For the deduction of the Mach cone equation far downstream of the obstacle $(x \rightarrow-\infty)$ it is more convenient to use a cylindrical $(x, \rho, \varphi)$ reference frame with clock angle $\varphi$ increasing from the $+Y$ direction (Fig. 1). In this reference frame:

$$
\begin{aligned}
& \boldsymbol{V}=V(-1,0,0) \quad \text { and } \\
& \boldsymbol{B}=B\left(-\cos \vartheta_{b v}, \sin \vartheta_{b v} \cos \varphi,-\sin \vartheta_{b v} \sin \varphi\right),
\end{aligned}
$$

and $\vartheta_{b v}$ range can be limited to $0 \leq \vartheta_{b v} \leq \pi / 2$ due to independence of MHD waves propagation velocity on the 
Table 1 .

\begin{tabular}{cccc}
\hline & condition & direction & $\sin \omega$ \\
\hline 1. & $M_{s} \ll M_{a} \rightarrow \infty$ & any & $\rightarrow 1 / M_{s}$ \\
2. & $M_{a} \ll M_{s} \rightarrow \infty$ & any & $\rightarrow 1 / M_{a}$ \\
3. & $\boldsymbol{B} \| \boldsymbol{V}$ & any & $\frac{\sqrt{M_{s}^{2}+M_{a}^{2}-1}}{M^{a} M^{s}}$ \\
4. & $\boldsymbol{B} \perp \boldsymbol{V}$ & $\pm \boldsymbol{B} \times \boldsymbol{V}$ & $\frac{\sqrt{M_{s}^{2}+M_{a}^{2}}}{M_{s} M_{a}}, \operatorname{maximal} \omega$ \\
5. & $\boldsymbol{B} \perp \boldsymbol{V}$ & $\pm \boldsymbol{B}$ & $\sqrt{\frac{M_{s}^{2}+M_{a}^{2}+1+\sqrt{\left(M_{s}^{2}+M_{a}^{2}+1\right)^{2}}-4 M_{s}^{2} M_{a}^{2}}{2 M_{s}^{2} M_{a}^{2}}}$ \\
6. & $\cos \vartheta_{b v}= \pm 1 / \min \left(M_{a}, M_{s}\right)$ & $\pm(\boldsymbol{B} \times \boldsymbol{V}) \times \boldsymbol{V}$ & $1 / \min \left(M_{a}, M_{s}\right), \operatorname{minimal} \omega$ \\
7. & $\sin \vartheta_{b v}=\frac{ \pm \sqrt{M_{s}^{2}+M_{a}^{2}}}{M^{s} M_{a}}$ & $\pm \boldsymbol{V} \times(\boldsymbol{B} \times \boldsymbol{V}), \operatorname{maximal} \omega$ & \\
\hline
\end{tabular}

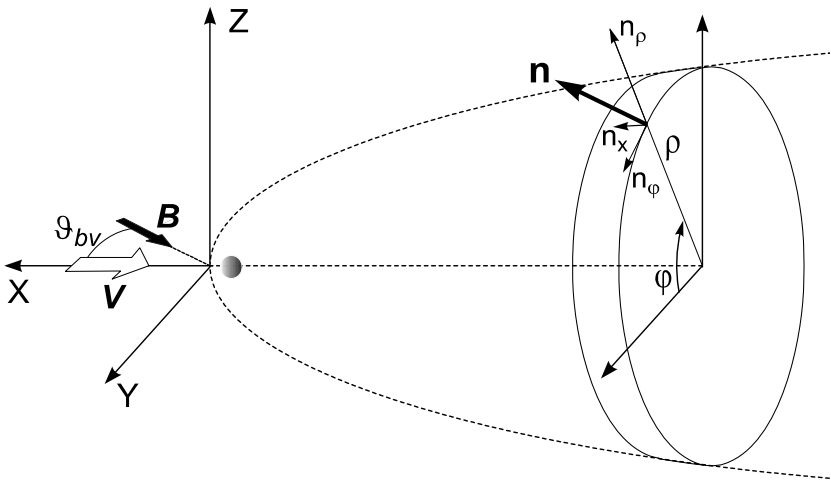

Fig. 1. Geometrical notations used for the MHD Mach cone determination.

magnetic field direction along the field line. The complicated bow shock surface $F(x, \rho, \varphi)=0$ (dashed curve in Fig. 1) will reduce to a simple conical boundary as $x \rightarrow-\infty$ :

$$
\left.F(x, \rho, \varphi)\right|_{x \rightarrow-\infty}=x+a(\varphi) \rho=0,
$$

with yet unknown $a(\varphi)$ function that determines $\varphi$ dependent radius $\rho$ of its cross-section at any $-x=$ const:

$$
\rho=\rho(\varphi)=\frac{-x}{a(\varphi)}=\frac{\text { const }}{a(\varphi)}
$$

and with $\varphi$-dependent asymptotic cone angle $\omega$ :

$$
\left.\tan \omega(\varphi)\right|_{\varphi=\text { const }}=-\frac{d \rho}{d x}=\frac{1}{a(\varphi)} .
$$

At any point on the shock surface all MHD RankineHugoniot conditions must be satisfied (Landau and Lifshitz, 1984):

$$
\begin{aligned}
& {\left[\rho_{p} V_{n}\right]=0, \quad\left[B_{n}\right]=0, \quad\left[B_{n} \boldsymbol{V}_{t}-V_{n} \boldsymbol{B}_{t}\right]=0,} \\
& {\left[\begin{array}{l}
\left.\rho_{p} V_{n}^{2}+p+\frac{\boldsymbol{B}_{t}^{2}-B_{n}^{2}}{8 \pi}\right]=0, \\
\left.\rho_{n} V_{n} \boldsymbol{V}_{t}-\frac{B_{n} \boldsymbol{B}_{t}}{4 \pi}\right]=0, \\
{\left[\rho_{p} V_{n} \frac{V^{2}}{2}+\frac{\gamma}{\gamma-1} V_{n} p+V_{n} \frac{B_{t}^{2}}{4 \pi}-\frac{B_{n}}{4 \pi}\left(\boldsymbol{V}_{t}, \boldsymbol{B}_{t}\right)\right]=0,}
\end{array}\right.}
\end{aligned}
$$

where brackets [ ] define the difference between the shock upstream and downstream bracketed expressions; $p, \rho_{p}$, and $\gamma$ are the pressure, mass density, and specific heats ratio of plasma flow, respectively, and indexes $n$ and $t$ mark the normal and tangential components of the vectors.

Solving relations (5) relative to the ratio of upstream and downstream plasma mass densities $\varepsilon$, one may deduce that this quantity must satisfy the following cubic equation at any point on the shock (e.g., see Petrinec and Russell, 1997):

$$
\begin{aligned}
(\gamma+ & 1) M_{a}^{6} \cos ^{6} \alpha_{v n} \cdot \varepsilon^{3} \\
& -M_{a}^{4} \cos ^{4} \alpha_{v n}\left((\gamma-1) M_{a}^{2} \cos ^{2} \alpha_{v n}\right. \\
& \left.+(\gamma+2) \cos ^{2} \vartheta_{b n}+\gamma+2 M_{a}^{2} / M_{s}^{2}\right) \cdot \varepsilon^{2} \\
& +M_{a}^{2} \cos ^{2} \alpha_{v n}\left(\left(\gamma-2+\gamma \cos ^{2} \vartheta_{b n}\right) M_{a}^{2} \cos ^{2} \alpha_{v n}\right. \\
& \left.+\left(1+\gamma+4 M_{a}^{2} / M_{s}^{2}\right) \cos ^{2} \vartheta_{b n}\right) \cdot \varepsilon \\
& -\left((\gamma-1) M_{a}^{2} \cos ^{2} \alpha_{v n}\right. \\
& \left.+2 M_{a}^{2} \cos ^{2} \vartheta_{b n} / M_{s}^{2}\right) \cos ^{2} \vartheta_{b n}=0
\end{aligned}
$$

where $\alpha_{v n}$ and $\vartheta_{b v}$ are the angles between the shock normal and upstream flow velocity $\boldsymbol{V}$ and magnetic field $\boldsymbol{B}$, respectively. Taking into account that $\varepsilon \rightarrow 1$ while $x \rightarrow-\infty$, the general equation (6) degenerates to the following relation applicable only far downstream of the obstacle:

$$
\begin{gathered}
\left(M_{a}^{2} \cos ^{2} \alpha_{v n}-\cos ^{2} \vartheta_{b n}\right) \cdot\left(\left(M_{a}^{2}+M_{s}^{2}\right) \cos ^{2} \alpha_{v n}\right. \\
\left.-M_{a}^{2} M_{s}^{2} \cos ^{4} \alpha_{v n}-\cos ^{2} \vartheta_{b n}\right)=0 .
\end{gathered}
$$

Setting the expression inside the first parenthesis in (7) describes the relation between $\alpha_{v n}$ and $\vartheta_{b n}$ for Alfvenic or rotational discontinuities while the second parenthesis corresponds to the same relationship at fast and slow MHD shocks (e.g., see paper by Kabin (2001) where equation for magnetoacoustic steady-state characteristics is presented in more symmetric shape).

Relation (2) provides the possibility to determine the shock normal $\boldsymbol{n}$ :

$$
\boldsymbol{n}=\frac{\nabla F}{|\nabla F|}=\frac{(1, a, \dot{a})}{\sqrt{1+a^{2}+\dot{a}^{2}}},
$$

where $\dot{a}=d a / d \varphi$. Taking into account relations (1), $\cos \alpha_{v n}$ and $\cos \vartheta_{b n}$ can be determined now as:

$$
\begin{aligned}
\cos \alpha_{v n} & =(\boldsymbol{V}, \boldsymbol{n}) / V=\frac{-1}{\sqrt{1+a^{2}+\dot{a}^{2}}}, \\
\cos \vartheta_{b n} & =(\boldsymbol{B}, \boldsymbol{n}) / B \\
= & \frac{-\cos \vartheta_{b v}+a \sin \vartheta_{b v} \cos \varphi-\dot{a} \sin \vartheta_{b v} \sin \varphi}{\sqrt{1+a^{2}+\dot{a}^{2}}}
\end{aligned}
$$




\subsection{Solution for the Alfven wings}

Substitution of relations (9) into the first parenthesis of relation (7) allows determining the equation for the Alfven wings:

$$
(\dot{a} \sin \varphi-a \cos \varphi) \sin \vartheta_{b v}+\cos \vartheta_{b v}= \pm M_{a} .
$$

General solution of this equation is straightforward:

$$
a(\varphi)=\frac{\cos \vartheta_{b v} \pm M_{a}}{\sin \vartheta_{b v}} \cdot \cos \varphi+k \sin \varphi,
$$

where $k$ is the integration constant. Than in the plane $-x=$ const $\rightarrow \infty$ :

$$
\rho(\varphi)=\frac{\text { const }}{\frac{\cos \vartheta_{b v} \pm M_{a}}{\sin \vartheta_{b v}} \cdot \cos \varphi+k \sin \varphi} .
$$

From Friedrichs I diagram for Alfven waves it follows that $\tan \omega=\rho /$ const $=0$ for $\varphi=\pi / 2,3 \pi / 2$. I.e., integration constant should be selected as $k \rightarrow \infty$. Then from relation (12) it follows that $\tan \omega=0$ in any direction except $\varphi=0$, $\pi$. Final expressions for Alfven wing slopes:

$$
\begin{aligned}
\tan \omega(0) & =\frac{\sin \vartheta_{b v}}{\cos \vartheta_{b v}+M_{a}}, \\
\tan \omega(\pi) & =\frac{\sin \vartheta_{b v}}{\cos \vartheta_{b v}-M_{a}},
\end{aligned}
$$

can easily be obtained also from Friedrichs II diagram for Alfven waves, which is degenerated into two points in the velocity space.

\subsection{Solution of Mach cone equation for the fast and slow MHD shocks}

Use of the second parentheses of relation (7) together with the relations (9) will lead to the equation for the asymptotic shock surface:

$$
\begin{gathered}
\left((\dot{a} \sin \varphi-a \cos \varphi) \sin \vartheta_{b v}+\cos \vartheta_{b v}\right)^{2} \\
=M_{a}^{2}+M_{s}^{2}-\frac{M_{a}^{2} M_{s}^{2}}{1+a^{2}+\dot{a}^{2}} .
\end{gathered}
$$

Once the nonlinear transcendental differential equation (14) is solved, the shape of the asymptotic bow shock crosssection $\rho(\varphi)$, as well as the shock cone angle $\omega(\varphi)$, can be simply determined via relations $(3,4)$, respectively.

Let us introduce two new functions:

$$
\begin{aligned}
& b(\varphi)=\dot{a} \sin \varphi-a \cos \varphi, \\
& c(\varphi)=\dot{a} \cos \varphi+a \sin \varphi,
\end{aligned}
$$

that are coupled to each other by the relation:

$$
\dot{c}(\varphi)=\dot{b}(\varphi) \cos \varphi / \sin \varphi .
$$

It also follows from definitions $(15,16)$ that:

$$
\begin{aligned}
& \dot{a}^{2}+a^{2}=b^{2}+c^{2}, \\
& a(\varphi)=-b \cos \varphi+c \sin \varphi .
\end{aligned}
$$

Substitution of $(15,18)$ into equation (14) immediately leads to relation:

$$
\left(b \sin \vartheta_{b v}+\cos \vartheta_{b v}\right)^{2}=M_{a}^{2}+M_{s}^{2}-\frac{M_{a}^{2} M_{s}^{2}}{1+b^{2}+c^{2}},
$$

that can simply be solved for $c(\varphi)$ :

$$
c^{2}=\frac{M_{a}^{2} M_{s}^{2}}{M_{a}^{2}+M_{s}^{2}-\left(b \sin \vartheta_{b v}+\cos \vartheta_{b v}\right)^{2}}-b^{2}-1 .
$$

Differentiation of relation (20) with subsequent use of coupling (17) leads to:

$$
\begin{aligned}
c & =\frac{\tan \varphi}{2} \frac{d c^{2}}{d b} \\
& =\tan \varphi \cdot\left(\frac{M_{a}^{2} M_{s}^{2}\left(b \sin \vartheta_{b v}+\cos \vartheta_{b v}\right) \sin \vartheta_{b v}}{\left(M_{a}^{2}+M_{s}^{2}-\left(b \sin \vartheta_{b v}+\cos \vartheta_{b v}\right)^{2}\right)^{2}}-b\right) .
\end{aligned}
$$

A final substitution of relation (21) into (20) will lead to an equation for the $b(\varphi)$ function:

$$
\begin{aligned}
\tan ^{2} \varphi & \cdot\left(\frac{M_{a}^{2} M_{s}^{2}\left(b \sin \vartheta_{b v}+\cos \vartheta_{b v}\right) \sin \vartheta_{b v}}{\left(M_{a}^{2}+M_{s}^{2}-\left(b \sin \vartheta_{b v}+\cos \vartheta_{b v}\right)^{2}\right)^{2}}-b\right)^{2} \\
& =\frac{M_{a}^{2} M_{s}^{2}}{M_{a}^{2}+M_{s}^{2}-\left(b \sin \vartheta_{b v}+\cos \vartheta_{b v}\right)^{2}}-b^{2}-1
\end{aligned}
$$

When this 10-th order polynomial non-differential (cf. equation (14)) equation is solved for $b(\varphi)$, we can find $c(\varphi)$ and $a(\varphi)$ from relations (21) and (19), respectively. This means that we have solved the problem and determined the Mach cone cross-section $\rho(\varphi)$ and cone angle $\omega(\varphi)$ that now can be calculated from relations $(3,4)$.

In fact, there is no need to solve equation $(22)$ for $b(\varphi)$ because the inverse function $\varphi(b)$ can be immediately written as:

$\varphi(b) \begin{cases}=\arctan \frac{\sqrt{\frac{M_{a}^{2} M_{s}^{2}}{M_{a}^{2}+M_{s}^{2}-\left(b \sin \vartheta_{b v}+\cos \vartheta_{b v}\right)^{2}}-b^{2}-1}}{\frac{M_{a}^{2} M_{s}^{2}\left(b \sin \vartheta_{b v}+\cos \vartheta_{b v} \sin \vartheta_{b v}\right.}{\left(M_{a}^{2}+M_{s}^{2}-\left(b \sin \vartheta_{b v}+\cos \vartheta_{b v}^{2}\right)^{2}\right.}-b} & \frac{d c^{2}(b)}{d b}>0, \\ =\pi-\arctan \frac{\sqrt{\frac{M_{a}^{2} M_{s}^{2}}{M_{a}^{2}+M_{s}^{2}-\left(b \sin \vartheta_{b v}+\cos \vartheta_{b v}\right)^{2}}-b^{2}-1}}{\frac{M_{a}^{2} M_{s}^{2}\left(b \sin \vartheta_{b v}+\cos \vartheta_{b v}\right) \sin \vartheta_{b v}}{\left(M_{a}^{2}+M_{s}^{2}-\left(b \sin \vartheta_{b v}+\cos \vartheta_{b v}\right)^{2}\right)^{2}}-b} & \frac{d c^{2}(b)}{d b}<0 .\end{cases}$

Proper plotting of the asymptotic cross-section of the shock requires preliminary study of equation (23). Figure 2 presents $c^{2}(b)$ function (20) between its vertical asymptotes located at:

$$
b=\frac{-\cos \vartheta_{b v} \pm \sqrt{M_{a}^{2}+M_{s}^{2}}}{\sin \vartheta_{b v}}
$$

There are generally four roots $b_{1-4}$ of $c^{2}(b)=0$ equation (Fig. 2). $\varphi(b)$ dependencies calculated with relations (23) for values of $b$ between the negative asymptote (24) and $b_{1}$, and between $b_{4}$ and the positive asymptote correspond to slow shocks. $\varphi(b)$ dependencies calculated for values of $b$ between $b_{2}$ and $b_{3}$ correspond to fast shocks.

Thus we have the possibility of constructing the inverse function $b(\varphi)$ for $0 \leq \varphi \leq \pi$ and, hence, $c(\varphi), a(\varphi), \rho(\varphi)$, and $\omega(\varphi)$ from relations $(21,19,3,4)$, respectively. Due to the fact that all boundaries have mirror symmetries relative to the $X, Y$ plane, $\omega(\varphi)=\omega(2 \pi-\varphi)$ and $\rho(\varphi)=\rho(2 \pi-\varphi)$ for the $\pi \leq \varphi \leq 2 \pi$ region. 


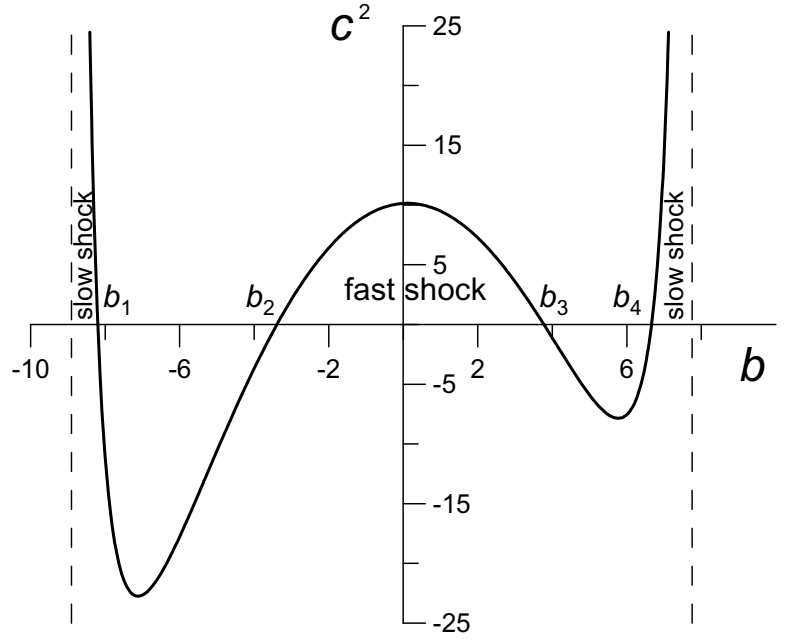

Fig. 2. $c^{2}(b)$ function between its vertical asymptotes for $M_{s}=6, M_{a}=4$, and $\vartheta_{b v}=60^{\circ}$.

\section{Results and Discussion of Specific Features}

Figure 3 graphs the tangent of the asymptotic slope $\omega$ for fast (smooth curve) and slow (two triangle-like features around $Y$ axis) shocks as functions of $\varphi$ normalized by $1 / \sqrt{\min \left(M_{a}^{2}, M_{s}^{2}\right)-1}$. The same curves can be considered as the proper shock cross-sections far downstream of the obstacle because of the proportionality of $\rho(\varphi)$ and $\tan \omega(\varphi)$ (see equations $(3,4))$. Two asterisks in Fig. 3 show similar characteristics for the Alfven wings in accordance with solutions (13).

The shape of the fast MHD shock cross-section is elongated in the $\pm Z$ direction and shifted in the $+Y$ direction. As expected (see Table 1), the asymptotic slope of the Mach cone is located between minimal and maximal ones. There is generally not any symmetry in the Mach cone cross-section except for the mirror symmetry relative to the $\boldsymbol{V}, \boldsymbol{B}$ plane $(Y$ axis in Fig. 3) and, of course, the additional mirror symmetry relative to the $Z$ axis for $\boldsymbol{B} \perp \boldsymbol{V}$ flow, or complete $X$ axial symmetry for $\boldsymbol{B} \| \boldsymbol{V}$ flow.

It can be checked directly that the present analytic solution $(23,21,19,3,4)$ contains all specific/symmetric cases tabulated in Table 1.

Both the shift in the $+Y$ direction and elongation in the $\pm Z$ direction of the fast MHD cone are connected to the increase of fast MHD wave propagation speed for decreasing cosine squared of the angle between the wave propagation direction $\boldsymbol{k}$ (shock normal $\boldsymbol{n}$ ) and magnetic field direction $\boldsymbol{B} / B$ as described by Friedrichs I diagram. In GIPM reference frame this cosine squared is generally smaller in the $+Y, \pm Z$ directions compared to the $-Y$ direction. The same arguments explain why the cross-section of the asymptotic slow MHD shocks and Alfven wings are shifted in the $-Y$ direction (Fig. 3): slow MHD and Alfvenic wave propagation speeds decrease with $(\boldsymbol{k}, \boldsymbol{B})^{2} / B^{2}$ decreasing.

The analytically deduced $+Y$ shift and $\pm Z$ elongated shape of the distant downstream fast shock cross-section qualitatively corresponds to the Earth's bow shock terminator cross-section shift and elongation as deduced by Peredo et al. (1995) after the analysis of a noisy set of 1392 bow

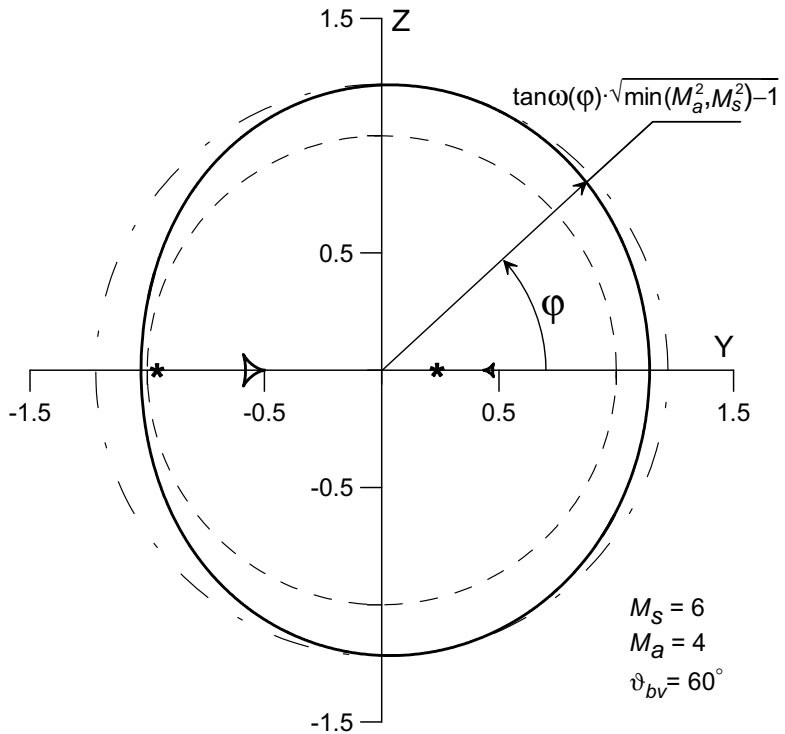

Fig. 3. Dependence on $\varphi$ of normalized tangent of the asymptotic slope $\omega$ of fast (smooth curve), and slow (triangle-like features) shocks, and of Alfven wings (asterisks). The same curves represents cross-section of these discontinuities far downstream of the obstacle. The dashed circle corresponds to the minimal possible $\omega$ of the fast shock and the dot-dashed circle to maximal (see Table 1).

shock observations by 17 orbiters, and by Verigin et al. (2001) after the analysis of a uniform set of 462 bow shock observations by the Wind orbiter with vectorial measurements of the upstream solar wind velocity.

Figure 4 presents the dependence on $M_{a}, M_{s}$ of the elongation of the asymptotic fast shock cross-section in the $\pm Z$ direction (upper panels) and shift in the $+Y$ direction (lower panels) calculated for $\vartheta_{b v}=30,60$, and $80 \mathrm{de}$ grees and $M_{m s}=M_{s} M_{a} / \sqrt{M_{s}^{2}+M_{a}^{2}}>1$ (smooth line in the panels). From the data presented in upper panels it is seen that the asymptotic fast shock cross-section elongation reaches its maximum when $M_{a}=M_{s}=M$. This corresponds to the maximal elongation of Friedrichs II dia$\operatorname{gram}\left(\min \left(M_{a}, M_{s}\right) / M_{m s}-1\right) \cdot 100 \%$ that is achieved when $M_{a}=M_{s}$ also. Maximal elongation at fixed $\vartheta_{b v}$ increases with $\vartheta_{b v}$ increasing: first the elongation is small $\leq 7.5 \%$ for $\vartheta_{b v} \leq 30^{\circ}$, then increases to $\sim 25 \%$ at $\vartheta_{b v}=60^{\circ}$, to $\sim 35 \%$ at $\vartheta_{b v}=80^{\circ}$, and approaches its maximum $(\sqrt{2}-1) \cdot 100 \% \approx$ $41 \%$ for $\vartheta_{b v}=90^{\circ}$ while $M \rightarrow \infty$ (this value of elongation is achieved at any $M$ for a Friedrichs II diagram).

The shift of the asymptotic fast shock cross-section in the $+Y$ direction also reaches a local maximum at $M_{a}=M_{s}=$ $M$ but has an additional tendency to increase while approaching small $M_{a}, M_{s}$ values. In spite of the elongation, the shift of the asymptotic fast shock cross-section approaches zero for both $\vartheta_{b v} \rightarrow 0^{\circ}$ and $\vartheta_{b v} \rightarrow 90^{\circ}$.

Figure 5 presents the variation of the fast shock asymptotic cross-section along the $M_{a}=M_{s}=M$ line in the $M_{a}, M_{s}$ plane (Fig. 4) for $\vartheta_{b v}=90^{\circ}$. The specific shape of the crosssection at higher $M$ reminds those one of 'kindersurprise' chocolate covered toys. Elongation of this cross-sections is high enough even for small and moderate Mach numbers. Thus just a simple rotation of the interplanetary magnetic field (without change of ram pressure, $M_{a}, M_{S}$ ) may lead to 

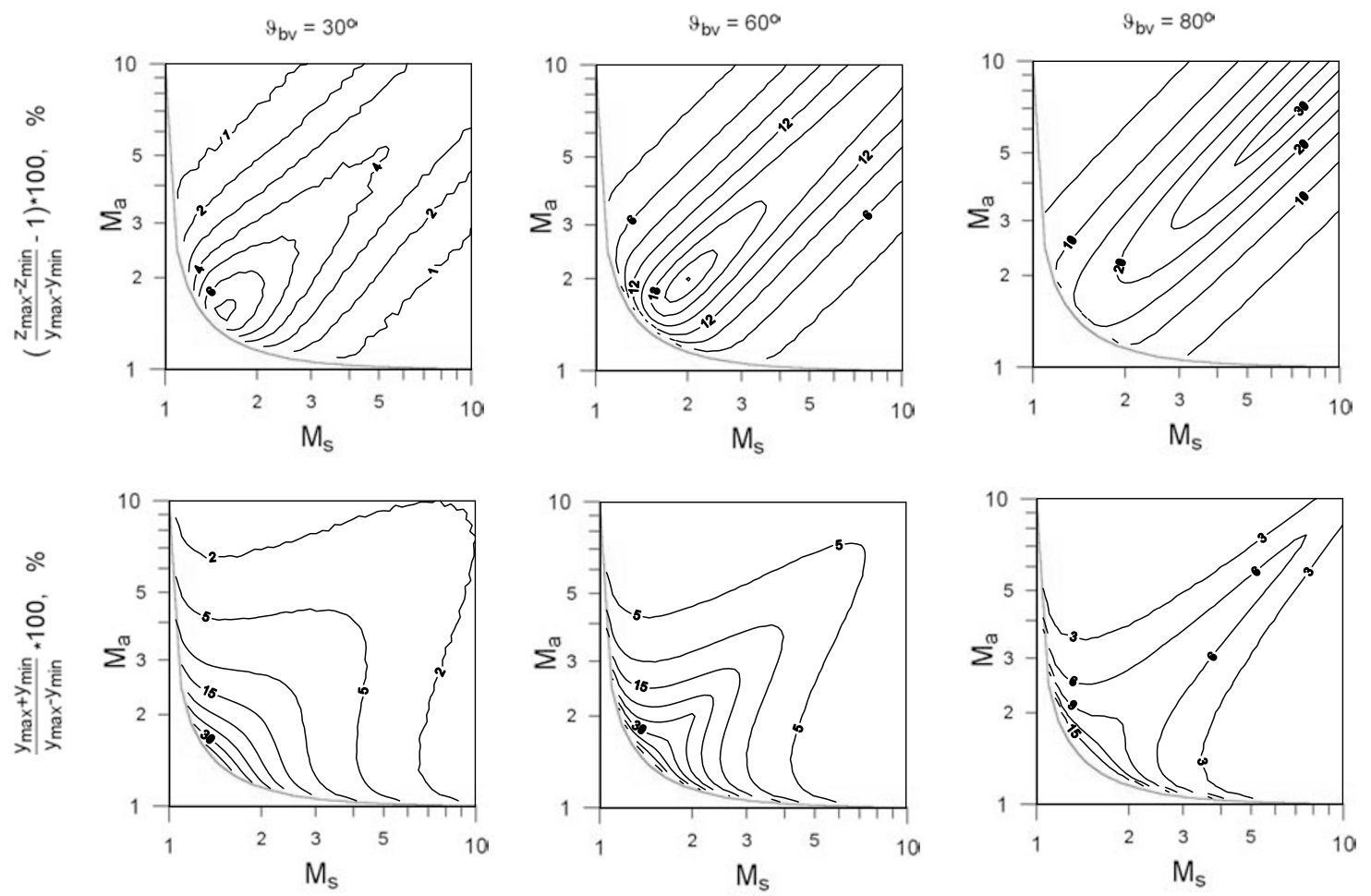

Fig. 4. Elongation in $\pm z$ direction of the asymptotic cross-section of fast shock (upper panels) and its shift in $+Y$ direction $\left(\right.$ lower panels) for $\vartheta_{b v}=30^{\circ}$, $60^{\circ}$, and $80^{\circ}$.
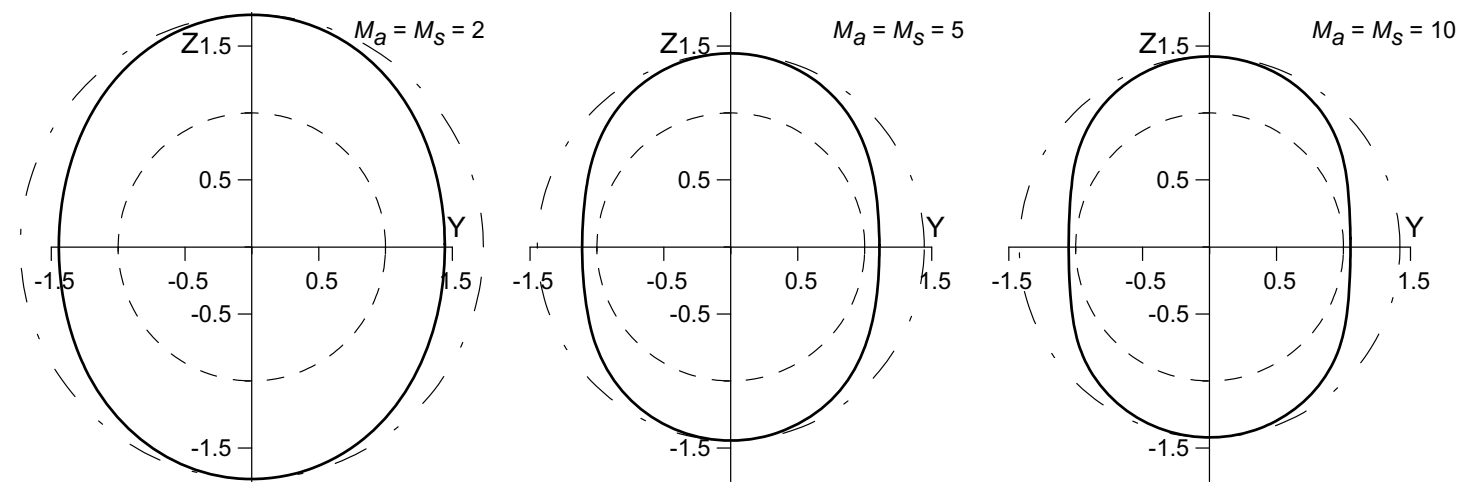

Fig. 5. Increase of elongation of asymptotic cross-section of 'kinder surprise' shape fast shock for $\vartheta_{b v}=90^{\circ}$ with increasing sonic and Alfvenic Mach numbers (from left to right). Dashed and dot-dashed circles correspond to those ones in Fig. 3.

a large displacement of the planetary downstream bow shock relative to an orbiter, and hence to the observation of multiple bow shock crossings.

It is worthwhile to note that shapes of these cross-sections generally do not correspond to the shapes of either Friedrichs I or Friedrichs II diagrams. The shape of the Friedrichs II diagram is the limiting case of the present solution in case $\vartheta_{b v}=90^{\circ}$ and $M_{a}, M_{s} \rightarrow \infty$ with some fixed $M_{a} / M_{s}$ ratio.

An especially interesting asymptotic fast shock crosssection, 'chopped' from the $-Y$ side is realized when $M_{a}=$ $M_{s}=M$ and $\cos \vartheta_{b v}= \pm 1 / M$ (Fig. 6). In this case the slope of the fast MHD Mach cone will achieve its minimal value in one direction (Table 1, line 6). Equality of $M_{a}$ and $M_{S}$ leads to the appearance of the 'cusp' feature in Friedrichs I diagrams (see e.g. figure 1 by Spreiter et al. (1966) paper) and to the appearance of the plane surface in a Friedrichs II diagrams, where both slow and fast magnetosonic group velocities are equal to each other (see e.g. figure 1 by Grad (1960) paper). In the case of $\cos \vartheta_{b v}= \pm 1 / M$ the crosssection of the sphere of $V / 2$ radius centered at the $-V / 2$ point with the Friedrichs I diagram is passing through the 'cusp', and the tangents to the Friedrichs II diagram are tracing through its plane region, thus providing appearance of the plane part of the asymptotic cross-section of the fast shock (Fig. 6).

Our solution with $M_{a}=M_{s}=M$ and $\cos \vartheta_{b v}= \pm 1 / M$ will lead to coincidence of the $b_{3}$ and $b_{4}$ roots of the $c^{2}(b)=$ 0 equation (Fig. 2). Substitution of $\cos \vartheta_{b v}= \pm 1 / M$, $\sin \vartheta_{b v}= \pm \sqrt{M^{2}-1} / M$ into equation (22) and its subsequent expansion into series in the vicinity of $b=\sqrt{M^{2}-1}$ 


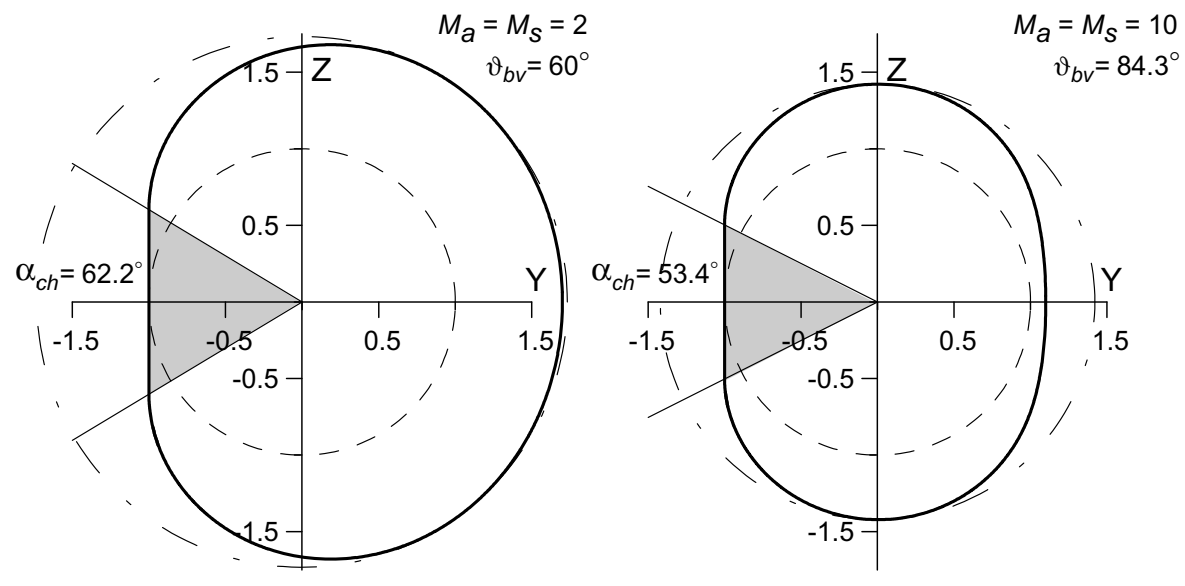

Fig. 6. Specific 'chopped' shape of the asymptotic cross-section of fast shock for $M_{a}=M_{s}=M$ and $\cos \vartheta_{b v}= \pm 1 / M$.

allows evaluation of the chopping angle $\alpha_{c h}$ (Fig. 6) of the asymptotic fast shock cross-section as:

$$
\alpha_{c h}=2 \arctan \left(\frac{M}{\sqrt{4 M^{2}-5}}\right) .
$$

The chopping angle is shaded in Fig. 6 in accordance with equation (25).

\section{Conclusions}

A direct approach to the determination of asymptotic MHD Mach cones is presented and applied to a range of upstream solar wind conditions. Our analytical solution provides the asymptotic downstream slope of Mach cones of MHD discontinuities for any clock angle and for arbitrary $M_{s}, M_{a}, \vartheta_{b v}$, and it recovers the previously observed shock asymmetries (Peredo et al., 1995; Verigin et al., 2001). An elongation and shift of the asymptotic fast shock crosssection was studied for a wide range of upstream plasma parameters as well as its unusual 'chopped' shape under certain conditions. The results obtained should be useful for planetary bow shock modeling and MHD numerical code verification.

Acknowledgments. This work was performed while one of the authors (MV) held a NRC Associateship Award at NASA/GSFC. Authors would like to thank Drs. D. H. Fairfield and K. Kabin for useful discussions.

\section{References}

Bennett, L., M. G. Kivelson, K. K. Khurana, L. A. Frank, and W. R. Paterson, A model of the Earth's distant bow shock, J. Geophys. Res., 102(A12), 26927-26941, 1997.

Grad, H., Reducible problems in magneto-fluid dynamic steady flows, Rev. of Modern Phys., 32(4), 830-847, 1960.

Jeffrey, A., Magnetohydrodynamics, Oliver \& Boyd ltd. publ., Edinburg, 1966.

Kabin, K., A note on the compression ratio in MHD shocks, J. Plasma Physics, 66(4), 259-274, 2001.

Khurana, K. K. and M. G. Kivelson, A variable cross-section model of the bow shock of Venus, J. Geophys. Res., 99(A5), 8505-8512, 1994

Landau, L. D. and E. M. Lifshitz, Electrodynamics of Continuous Media, Pergamon publ., Oxford, 1984

Peredo, M., J. A. Slavin, E. Mazur, and S. A. Curtis, Three-dimensional position and shape of the bow shock and their variation with Alfvenic, sonic and magnetosonic Mach numbers and interplanetary magnetic field orientation, J. Geophys. Res., 100, 7907-7916, 1995.

Petrinec, S. M. and C. T. Russell, Hydrodynamic and MHD equations across the bow shock and along the surfaces of planetary obstacles, Space Sci. Rev., 79, 757-791, 1997.

Slavin, J. A., R. E. Holzer, J. R. Spreiter, and S. S. Stahara, Planetary Mach cones: Theory and observation, J. Geophys. Res., 89(A5), 2708-2714, 1984.

Spreiter, J. R. and S. S. Stahara, Magnetohydrodynamic and gasdynamic theories for planetary bow waves, in Collisionless Shocks in the Heliosphere: Reviews of Current Research, edited by by B. T. Tsurutany and R. G. Stone, pp. 85-107, Geophysical Monograph series No. 35, AGU publ., Washington, D.C., 1985.

Spreiter, J. R., A. L. Summers, and A. Y. Alksne, Hydromagnetic flow around the magnetosphere, Planet. Space Sci., 14, 223-253, 1966.

Verigin, M. I., G. A. Kotova, A. P. Remizov, V. A. Styazhkin, N. M. Shutte, T.-L. Zhang, W. Riedler, H. Rosenbauer, K. Szego, M. Tatrallyay, and K. Schwingenschuh, Shape and location of planetary bow shocks, Cosmic Research, 37(1), 34-39, 1999.

Verigin, M., G. Kotova, A. Szabo, J. Slavin, T. Gombosi, K. Kabin, F. Shugaev, and A. Kalinchenko, Wind observations of the terrestrial bow shock: 3-D shape and motion, Earth Planets Space, 53(10), 1001-1009, 2001 .

Whitham, G. B., Linear and nonlinear waves, A Wiley-Interscience publ., New York, 1974

M. Verigin (e-mail: verigin@iki.rssi.ru), J. Slavin, A. Szabo, G. Kotova, and T. Gombosi 